\title{
Data Mining of Free-Text Responses: An Innovative Approach to Analyzing Patient Perspectives on Treatment for Chronic Rhinosinusitis with Nasal Polyps in a Phase lla Proof-of-Concept Study for Dupilumab
}

\author{
Asif $\mathrm{H}$ Khan (D) \\ Adeline Abbe' \\ Bruno Falissard ${ }^{2}$ \\ Paulo Carita' \\ Claus Bachert ${ }^{3,4}$ \\ Joaquim Mullol (D) ${ }^{5}$ \\ Matthew Reaney ${ }^{6}$ \\ Jingdong $\mathrm{Chao}^{7}$ \\ Leda P Mannent ${ }^{1}$ \\ Nikhil Amin ${ }^{7}$ \\ Puneet Mahajan ${ }^{8}$ \\ Gianluca Pirozzi ${ }^{8}$ \\ Laurent Eckert ${ }^{\prime}$ \\ 'Sanofi, Chilly-Mazarin, France; ${ }^{2}$ Centre de \\ recherche en epidémiologie et santé des \\ populations (CESP), INSERM UI0I8, Paris, \\ France; ${ }^{3}$ Upper Airways Research \\ Laboratory, Ghent University, Ghent. \\ Belgium; ${ }^{4}$ CLINTEC, Karolinska Institutet, \\ Stockholm, Sweden; ${ }^{5}$ Rhinology Unit \& \\ Smell Clinic, ENT Department, Hospital \\ Clínic, Universitat de Barcelona; Clinical \\ and Experimental Respiratory \\ Immunoallergy, IDIBAPS; and CIBERES, \\ Barcelona, Catalonia, Spain; ' ${ }^{6} \mathrm{QVIA}$, \\ Reading, UK; ${ }^{7}$ Regeneron Pharmaceuticals, \\ Inc., Tarrytown, NY, USA; ${ }^{8}$ Sanofi, \\ Bridgewater, NJ, USA
}

Correspondence: Asif $\mathrm{H}$ Khan Sanofi, I Avenue Pierre Brossolette, Chilly-Mazarin, 91380, France Tel +33 I 60497777

Email asif.khan@sanofi.com
Purpose: Patient perspective is an important and increasingly sought-after complement to clinical assessment. The aim of this study was to transcribe individual patients' experience of treatment in a dupilumab clinical trial through free-text responses with analysis using natural language processing (NLP) to obtain the unique perspective of patients on disease impact and unmet needs with existing treatment to inform future trial design.

Patients and Methods: Patients with chronic rhinosinusitis with nasal polyps (CRSwNP) who were enrolled in a Phase IIa randomized controlled trial comparing dupilumab with placebo (NCT01920893) were invited to complete a self-assessment of treatment (SAT) tool at the end of treatment, asking, "What is your opinion on the treatment you had during the trial? What did you like or dislike about the treatment?" Free-text responses were analyzed for the overall cohort and according to treatment assignment using natural language processing including sentiment scoring. In a mixed-methods approach, quantitative patient-reported outcome (PRO) results were utilized to complement the qualitative analysis of free-text responses.

Results: Of 60 patients enrolled in the study, 43 (71.6\%) completed the SAT and responses from 37 patients were analyzed (placebo, $n=16$; dupilumab, $n=21$ ). Word analyses showed that the most common words were "smell," "improve," "staff," "great," "time," and "good." Across the whole cohort, "smell" was the most common symptom-related word. The words "smell" and "experience" were more likely to occur in patients treated with dupilumab. Patients treated with dupilumab also had more positive sentiment in their SAT responses than those who received placebo. The results from this qualitative analysis were reflected in quantitative PRO results.

Conclusion: "Smell" was important to patients with CRSwNP, highlighting its importance as a patient-centric efficacy outcome measure in the context of clinical trials in CRSwNP.

Trial Registration: ClinicalTrials.gov, NCT01920893. Registered 12 August 2013, https:// www.clinicaltrials.gov/ct2/show/NCT01920893.

Keywords: CRSwNP, free-text data mining, patient perspective, self-assessment, sense of smell

\section{Introduction}

To comprehensively understand illness and the effects of treatment, it is necessary to supplement clinical, radiologic, laboratory, and other evidence reported by clinicians with the patient's own perspective. Together, these elements support 
a fundamental role of medical science: "to transcribe the individual patient's experience of illness; to make a doctor's story out of the patient's own". ${ }^{1}$ Each patient has their own narrative of their illness and constructive engagement with this narrative, termed "narrative medicine", may uncover valuable diagnostic and therapeutic options. $^{2}$ In the context of clinical trials, patient experiences, perspectives, needs, and priorities are increasingly sought by healthcare regulators, payers, and physicians. ${ }^{3,4}$ The patient's perspective can assist in determining disease impacts, identifying unmet medical needs for existing and new treatments, informing new subpopulations and targets for drug discovery, and evaluating endpoints of relevance to patients. ${ }^{4}$ Recent guidance recommends that patient experiences and qualitative research should be incorporated early in the drug development process, ${ }^{5}$ and the use of patientreported outcome measures (PROMs) is increasing. ${ }^{6}$

Patient satisfaction is one type of PROM; it refers to the patient's evaluation of the process of taking the medication and the outcomes associated with the medication. ${ }^{7}$ While PROMs have been developed and validated to quantitatively describe patients' treatment satisfaction, ${ }^{8}$ the concept is complex, subjective, and inherently heterogeneous, and can benefit from capture and analyses of unstructured responses to questions. ${ }^{9}$ Allowing the patient to give unstructured responses to questions without the constraints of closed questions not only builds awareness and insight of patient perspectives on disease and treatment, but is also potentially complementary to quantitative PROM data by providing information on experiences not captured using these tools. ${ }^{6,10}$ Methods that combine both quantitative and qualitative approaches have not often been utilized in clinical trials.

Mining patients' "free-text" responses to open questions is an innovative approach to free-text analysis that provides relevant, interpretable, and actionable information ${ }^{10}$ on patient experience. In natural language processing (NLP), algorithms are utilized to derive patterns within the textual data, to allow for evaluation and interpretation of findings. The approach has been used successfully to explore the patient voice in atopic dermatitis, cancer, and psychiatry research. ${ }^{6,11,12}$

Chronic rhinosinusitis with nasal polyps (CRSwNP) is a chronic inflammatory disease of the nasal and paranasal sinuses that is associated with a high symptom and economic burden and poor health-related quality of life
(HRQoL). ${ }^{13-17}$ CRSwNP predominantly displays a type 2 inflammatory signature, which is associated with the prominent expression of the cytokines interleukin (IL)-4, IL-5, and IL-13. ${ }^{18,19}$ Information on patient perspective of CRSwNP treatment during a randomized clinical trial is lacking.

Dupilumab, a fully human VelocImmune ${ }^{\circledR}$-derived ${ }^{20,21}$ monoclonal antibody, blocks the shared receptor component for IL-4 and IL-13, cytokines that are key and central drivers of type 2 diseases such as atopic dermatitis, asthma, CRSwNP, allergic rhinitis, and food allergies, which are often associated as comorbidities, ${ }^{22}$ thus inhibiting their signaling. Dupilumab is approved for patients with type 2 inflammatory diseases, including atopic dermatitis, asthma, and CRSwNP. ${ }^{23,24}$

In the Phase IIa proof-of-concept study (NCT01920893) and in two Phase III studies (NCT02912468; NCT0289454) in patients with CRSwNP refractory to intranasal corticosteroids, dupilumab on a background of mometasone furoate nasal spray (MFNS) significantly improved both subjective and objective assessments, including symptoms such as sense of smell, endoscopic nasal polyp (NP) score, and computed tomography (CT) scan score, respectively. ${ }^{25,26}$ Dupilumab also showed improvement in PROMs including disease severity and HRQoL. ${ }^{27}$

The objectives of the present study were to (i) explore the feasibility of capturing patient narratives of illness and treatment in a dupilumab clinical trial in CRSwNP using free-text responses and (ii) investigate the potential of transcribing these narratives using natural language processing (NLP) to gain insights to inform the design of future clinical trials of treatments for CRSwNP. Such insights might include the identification of subgroups of patients with high unmet needs, or outcomes most important to patients in this study population, which has different selection criteria and thus different characteristics from the populations used in the development of validated PROMs. A mixed-methods approach was utilized, in which the qualitative analysis of free-text responses was complemented with quantitative PROM results.

\section{Patients and Methods}

\section{Population}

This analysis was based on a Phase IIa, multicenter, randomized, double-blind, placebo-controlled, parallelgroup study in 60 adults with CRSwNP, details of which are reported elsewhere. ${ }^{25}$ Briefly, patients were assigned 1:1 to add-on therapy with subcutaneous 
dupilumab (a $600 \mathrm{mg}$ loading dose followed by $300 \mathrm{mg}$ once weekly for 16 weeks) or matched placebo, on a background of MFNS.

\section{Data Collection}

As part of the study, patients had the option of completing a voluntary self-assessment of treatment (SAT) tool at the end of treatment. Patients were asked to respond to an open-ended question with a free-text input that asked, "What is your opinion on the treatment you had during the trial? What did you like or dislike about the treatment?" The trial was conducted in the USA, the Netherlands, the UK, Sweden, and Spain, and patients provided a handwritten response to the SAT in their native language. Responses in Dutch, Swedish, and Spanish were digitized, transcribed, and linguistically translated into English using forward and backward translations. Responses missing the anonymized Subject ID were not analyzed, as treatment arm could not be determined.

\section{Analyses}

\section{Natural Language Processing}

Responses to the SAT were analyzed using NLP, the process of automated extraction and structuring of text to identify patterns and derive meaningful conclusions from unstructured text through analysis of word occurrence, frequency, and linguistic features. ${ }^{28}$ Analysis was performed on the translated dataset.

The frequencies of specific words were tabulated and word clouds constructed for the overall population and the two treatment arms (dupilumab, placebo) to derive a visual representation of the most frequently reported words, in which font size within the cloud indicates word frequency. ${ }^{29}$ In order to avoid visual noise, the word "trial" was removed, and only words appearing at least twice were included.

Co-occurrence identified words with the highest degree of centrality. The list of co-occurrences was then ordered by decreasing frequency to construct a network (using $\mathrm{R}$ software). Using this network, the number of subcommunities and words belonging to each subgroup were determined. Finally, community detection was used to define a "natural" division of a network into groups of nodes using the Fastgreedy algorithm..$^{30,31}$

Sentiment analysis, which helps determine overall sentiment in terms of emotions or opinions expressed within an unstructured piece of text, was used to enable a deeper understanding of the attitudes of a person with respect to a topic in a piece of text. Participants' sentiments of their treatment experience were determined by matching individual words to their sentiment scores, which was provided by an external sentiment reference dataset. Sentiment labeling as positive, neutral, or negative was performed manually for all available samples. Sentiment analysis was performed for the following outcomes: participants' feelings about their medication (Medication); participants' feelings about their treatment (Treatment); and participants' overall experience (Overall experience).

\section{Disease Outcome Measures}

The 22-item Sino-Nasal Outcome Test (SNOT-22) score for assessment of impact on HRQoL and University of Pennsylvania Smell Identification Test (UPSIT) score, and daily patient-reported loss of smell score for assessment of impact on loss of sense of smell, were evaluated in the subgroup of patients who completed the SAT and included their Subject ID (for whom treatment allocation could be assigned) and for the subgroup who did not complete the SAT or did not provide Subject ID. PROMs were analyzed using a mixed-effect model with repeated measures (MMRM) approach as described previously. ${ }^{25}$ The model included change from baseline as response variable with fixed-effects factors for treatment, stratification (comorbid asthma, biopsy performed), visit, treatment by visit interaction, nasal polyp score baseline value, and baseline by visit interaction. The model did not impute missing data points.

\section{Results \\ Dataset}

Of 60 patients enrolled in the Phase IIa study, 43 $(71.6 \%)$ completed the voluntary SAT, and responses from 37 patients were analyzed (placebo, $n=16$; dupilumab, $\mathrm{n}=21$; six responses were not analyzed due to missing Subject ID). SATs were completed in Dutch $(\mathrm{n}=13)$, English $(\mathrm{n}=11)$, Spanish $(\mathrm{n}=14)$, and Swedish $(\mathrm{n}=5)$. Text responses varied between four and 226 words. Demographics and baseline clinical characteristics are summarized in Table 1 . The treatment groups were generally well matched at baseline, although patients assigned to placebo had a longer mean duration of disease than those assigned to dupilumab. Patients who did not complete the SAT had worse baseline UPSIT and loss of smell score than patients who completed the SAT. 
Table I Baseline Demographics and Clinical Characteristics

\begin{tabular}{|c|c|c|c|c|}
\hline & \multicolumn{2}{|c|}{ Patients Who Completed the SAT } & \multicolumn{2}{|c|}{$\begin{array}{c}\text { Patients Who Did Not Complete } \\
\text { the SAT }\end{array}$} \\
\hline & $\begin{array}{l}\text { Placebo/MFNS } \\
\qquad(n=16)\end{array}$ & $\begin{array}{c}\text { Dupilumab/ } \\
\text { MFNS }(n=2 I)\end{array}$ & $\begin{array}{l}\text { Placebo/MFNS } \\
\qquad(n=14)\end{array}$ & $\begin{array}{l}\text { Dupilumab/ } \\
\text { MFNS }(n=9)\end{array}$ \\
\hline Age, mean (SD), years & $50.4(10.5)$ & $46.4(8.5)$ & $48.0(7.4)$ & $49.9(12.4)$ \\
\hline Male sex, n (\%) & $8(50.0)$ & $13(61.9)$ & $8(57.1)$ & $5(55.6)$ \\
\hline NP duration, mean (SD), years & $10.2(8.0)$ & $7.5(5.6)$ & $12.9(9.5)$ & $7.8(7.7)$ \\
\hline Bilateral endoscopic NPS, mean (SD), range $0-8^{a}$ & $5.8(0.7)$ & $6.0(0.7)$ & $5.6(1.1)$ & $5.7(1.5)$ \\
\hline Lund-MacKay total CT score, mean (SD), range $0-24^{a}$ & $18.4(4.6)$ & $19.0(4.5)$ & $19.1(6.6)$ & $17.8(6.3)$ \\
\hline Smell test (UPSIT) score, mean (SD), range $0-40^{\mathrm{b}}$ & $13.9(4.5)$ & $11.8(7.0)$ & $17.6(10.4)$ & I5.I (10.8) \\
\hline Sense of smell loss, mean (SD), range $0-3^{\mathrm{a}, \mathrm{c}}$ & $2.9(0.3)$ & $2.5(0.9)$ & $2.7(0.7)$ & $2.2(I . I)$ \\
\hline SNOT-22 total score, mean (SD), range $0-110^{\mathrm{a}}$ & $4 I . I(22.2)$ & $43.1(19.8)$ & $39.9(17.7)$ & $37.6(14.1)$ \\
\hline CRSwNP disease severity (VAS), mean (SD), range $0-10 \mathrm{~cm}^{\mathrm{a}}$ & $5.4(3.1)$ & $6.3(2.5)$ & $7.7(1.5)$ & $6.7(3.4)$ \\
\hline Patients with comorbid asthma, $\mathrm{n}(\%)$ & $10(62.5)$ & $13(61.9)$ & $9(64.3)$ & $3(33.3)$ \\
\hline Patients with comorbid NSAID-ERD, n (\%) & $5(3 \mid .3)$ & $6(28.6)$ & $5(35.7)$ & I (II.I) \\
\hline
\end{tabular}

Notes: ${ }^{a}$ Higher scores indicate worse status. ${ }^{b}$ Higher scores indicate better status. ${ }^{c} 0=$ no symptoms, $\mathrm{I}=$ mild symptoms, $2=$ moderate symptoms, $3=$ severe symptoms. Abbreviations: CRSwNP, chronic rhinosinusitis with nasal polyps; CT, computed tomography; MFNS, mometasone furoate nasal spray; NP, nasal polyposis; NPS, nasal polyp score; NSAID-ERD, non-steroidal anti-inflammatory drug-exacerbated respiratory disease; SAT, self-assessment of treatment; SD, standard deviation; SNOT-22, 22item Sino-Nasal Outcome Test; UPSIT, University of Pennsylvania Smell Identification Test; VAS, Visual Analog Scale.

\section{Word Clouds}

Construction of a word cloud based on word frequency is shown for the overall cohort of 37 patients who completed the SAT in Supplementary Figure 1. Words that occurred at high frequency included "smell," "improve," "staff," "great," "time," and "good." Word clouds based on word frequency were also constructed for completers who were assigned to dupilumab (Figure 1A) or placebo (Figure 1B). The word cloud for dupilumab differed from that for placebo. In the dupilumab arm, the most frequent words to follow "good" were "smell" and "not." In the placebo arm, in contrast, the most frequent words to follow "good" were "not" and "improve."

\section{Word Co-Occurrences}

Word clusters reporting the co-occurrences of words used by patients treated with dupilumab or placebo are shown in Figure 2. These word clusters differed between dupilumab and placebo. In the dupilumab arm, examples of words that co-occurred were "smell, taste, sense, study, satisfy, problem, part," "experience, effect," and "polyp, breathe, improve, help, nose, runny, hope, shot, feel, drug." In the placebo arm, examples of words that co- occurred were "treatment, staff," "study, part," "great, shot," "breathe, nose, trial, test," and "good, professional, feel, sense, smell, time." The context of these co-occurring words can be seen in samples of full-text responses from individual patients (Supplementary Table 1).

\section{Sentiment Analysis}

Participants' feelings related to their medication, how they were treated during the study, and their overall experience were explored in sentiment analysis in the overall cohort of SAT completers, and in patients assigned to placebo and dupilumab (Figure 3). More instances of positive sentiment were recorded for each domain in patients treated with dupilumab compared with placebo. The percentage of patients expressing negative sentiment in relation to medication was especially apparent in placebo-treated patients.

\section{Disease Outcome Measures}

PROMs including loss of smell and HRQoL in the group of patients who completed the SAT are shown in Supplementary Figure 2. At Week 16, patients treated with dupilumab compared with placebo had greater improvement in SNOT-22 total score (least squares mean 
A

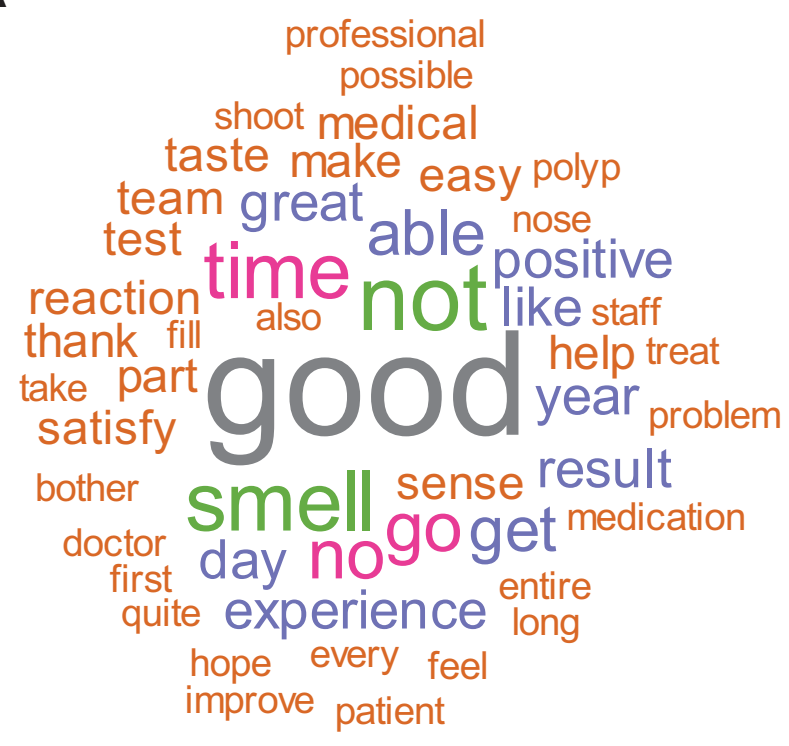

B

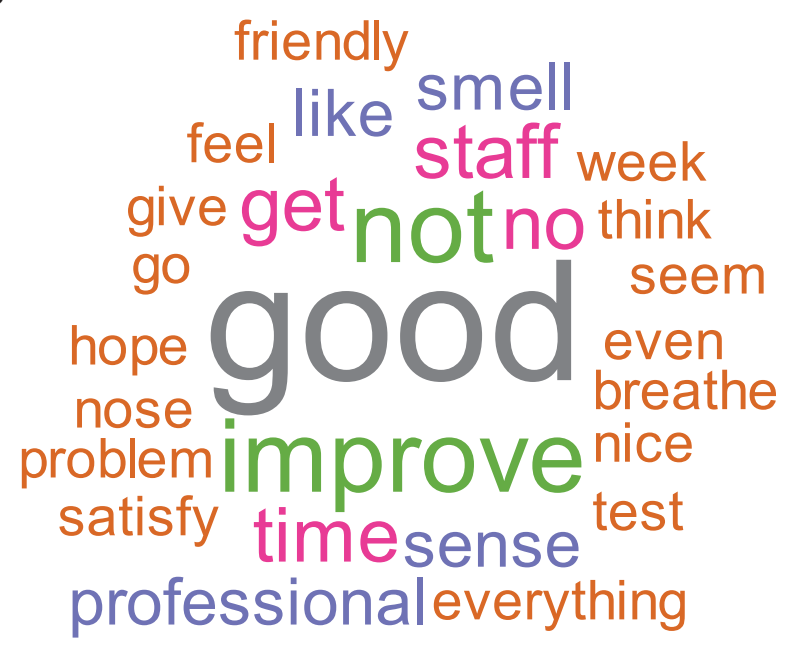

Figure I Word clouds in patients treated with (A) dupilumab or (B) placebo. Word cloud based on frequency (higher frequency = bigger font size). Only words that occur twice or more are shown. Stop words and the words "study" and "treatment" are omitted.

difference versus placebo [95\% confidence interval]: -20.75 [-29.64, -11.85$] ; p<0.0001)$, UPSIT score (14.50 [9.61, 19.39]; $p<0.0001)$, and loss of smell score $(-1.35[-1.94,-0.77] ; p<0.0001)$. Significant improvements with dupilumab versus placebo were also seen for UPSIT and loss of smell score in patients who did not complete the SAT, despite the small subgroup size $(n=23)$ (Supplementary Table 2). SNOT-22 score also improved with dupilumab versus placebo in patients who did not complete the SAT, but the difference did not reach statistical significance.

\section{Discussion}

In CRSwNP, the burden of disease and value of interventions have previously been investigated from the patient perspective using PROMs. ${ }^{25,32,33}$ Studies measuring HRQoL in patients with CRSwNP have reported improvements in subjective outcomes following surgical intervention $^{33}$ and drug therapy. ${ }^{33}$ However, no randomized studies involving patients with CRSwNP have used NLP to glean clinically important meanings from free-text patient narratives obtained within clinical trials. Free-text narratives allow patients to give unrestricted feedback without the constraints of structured instruments and are complementary to quantitative PROM data. Analysis of free-text responses using NLP has the potential to provide meaningful conclusions about experiences that are specific to the target population, and which may not be captured using traditional PROM questionnaires, which use "forced choice" answers.

Free text data analysis is increasingly used to evaluate patient perception, experience, and satisfaction with care delivery. ${ }^{34,35}$ The approach presented here to capture and analyze free-text responses in the setting of a clinical trial illustrates the insights that can be achieved to identify subpopulations with high unmet medical needs, targets for treatment, and outcomes that are of importance to patients for inclusion in clinical trials. To our knowledge, this is the first study to investigate free-text responses in the context of a clinical trial in patients with CRSwNP. More than $70 \%$ of patients provided a response on the SAT, showing the feasibility of this method for capturing patient experience. This approach also introduces an element of "narrative medicine" into the clinical trial process, which as well as being patientcentric, provides a window on the personal connections between patient and treatment that may not otherwise be captured and may help inform aspects of future trial design, such as subpopulations of patients with high unmet medical needs or the hierarchy of PROMS.

The present analysis provided patient "bedside feedback" on patients' experience of CRSwNP treatments by evaluating the frequency with which words occurred in the free-text responses. "Smell" was the most common symptom-related word in the word cloud analysis, indicating the importance of smell to patients with CRSwNP, and its centrality, both in terms of how patients perceive the disease burden and the improvement in symptoms resulting from dupilumab therapy. The importance of smell perception is illustrated in the free-text response 


\section{A}

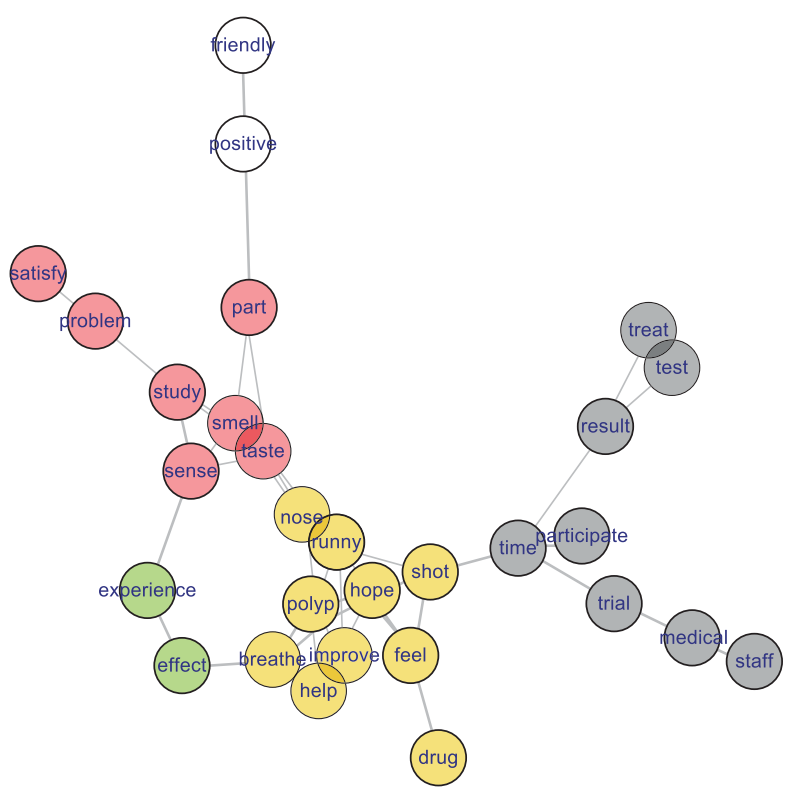

B
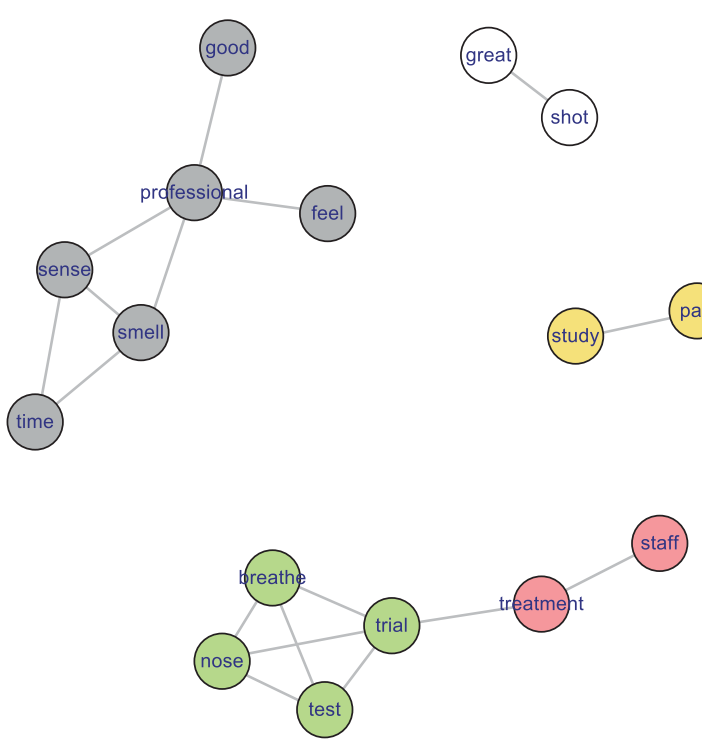

Figure 2 Co-occurrence of words in patients completing a self-assessment tool treated with (A) dupilumab, (B) placebo. Words in the same cluster were more frequently reported together. Colors identify word clusters.

"I just hope and pray that my smell and taste lasts a long time. I love being able to smell my sweet two year old and my yummy food $\odot$. Thanks a million!". In particular, the findings show that patients treated with dupilumab emphasized the improvement in smell and breathing. Patients treated with placebo, in contrast, were hopeful of smell improvement, which further reinforces the importance of this symptom for CRSwNP patients, and also more often expressed the care they received from medical personnel during the study.
The PROM instruments were scheduled for completion at study visits before any procedure and we cannot be certain of the sequence in which each individual patient completed the instruments. The potential for the SAT responses to be influenced by the PROM instruments was limited by the SAT being administered at the last visit and asking the patient to provide an overall impression of the treatment. The clear signal indicating the importance of smell in the SAT emerged despite smell being a relatively minor part of the PROM items. The 


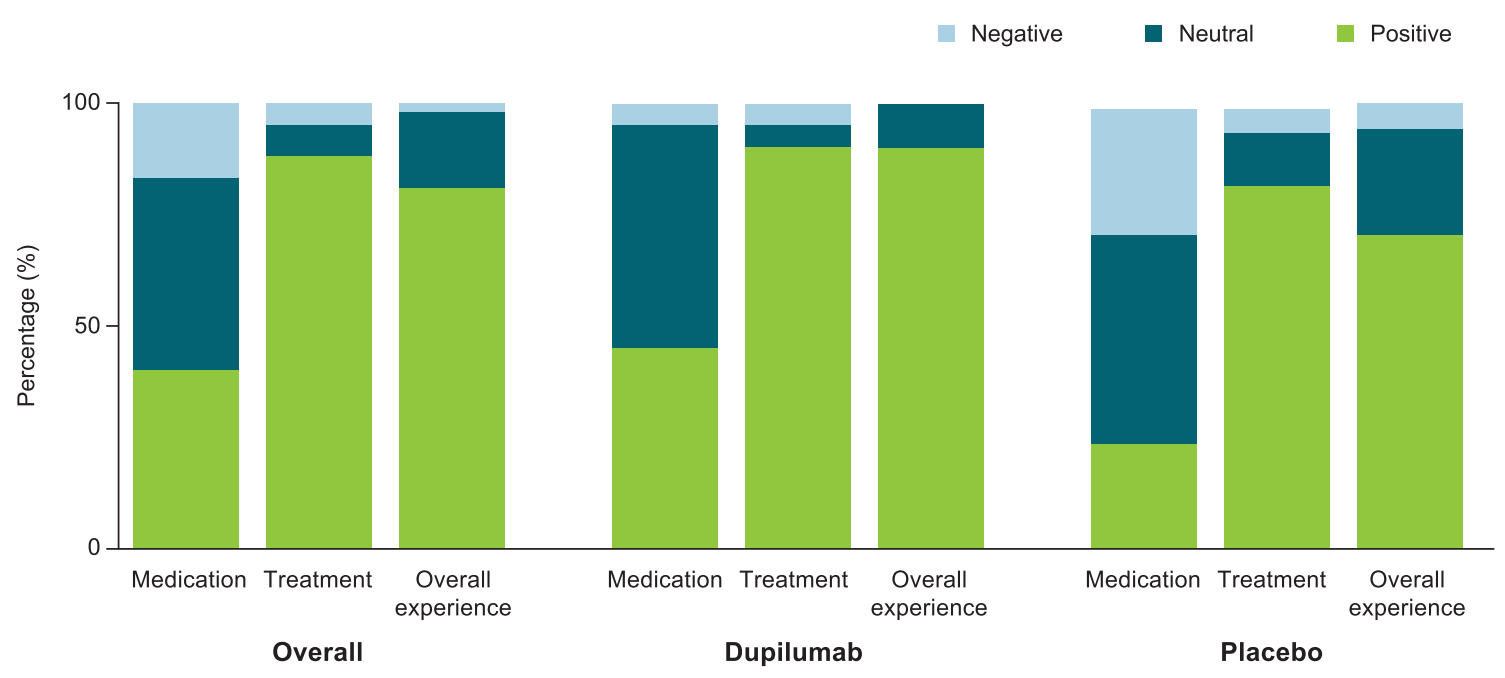

Figure 3 Sentiment analysis of patients completing a self-assessment tool: overall, dupilumab group, and placebo group.

negative influence of olfactory disorders on HRQoL has previously been highlighted using PROs. ${ }^{36-38}$ Further to the NLP analysis in this study, PROs including loss of smell and HRQoL reflected the findings of the text-mining approach. Patients treated with dupilumab, who demonstrated a qualitative improvement in smell and breathing in the SAT, had significantly greater improvements in patientreported sense of smell and HRQoL than patients receiving placebo, who reported hope of smell improvement in the SAT. These findings support the value of utilizing a mixed-methods approach.

The present findings complement these earlier findings in providing qualitative support for the value that patients place on their sense perception and the impact its loss has on HRQoL. Word co-occurrences highlighted the different patient perspectives based on treatment assignment. With dupilumab, words related to sense perception had frequent co-occurrences with words such as "improvement" and "better" as in "I can smell and taste better than I have in the past 10 years." Although patients who were assigned to placebo also expressed words related to sense perception, these words were more likely to co-occur with "hope" as in "I hope that if the medicine comes on the market that I will be eligible for it" and "satisfied" as in "I am very satisfied with how the study went." From a general perspective, this self-assessment of treatment of CRSwNP using NLP allowed patients to respond without predefined choices, which are subject to possible investigator bias ${ }^{10}$ and do not generate the kind of in-depth data that may be gleaned from patient narratives. ${ }^{39}$ The text-mining approach in the present context provided this in-depth insight into patients' treatment perspectives, and in particular provided patient-level evidence for smell as an important symptom for NP. NLP is thus an invaluable method for understanding how patients comprehend the impact of disease and its treatment on HRQoL. ${ }^{40,41}$ Having confirmed the importance of sense of smell perception from a patient perspective, an immediate application of this finding will ensure that sense of smell is adequately captured as a PRO measure in future studies of patients with CRSwNP. The approach used in the current analysis could be valuable for the evaluation of patient perspectives in other disease areas and to identify unmet medical needs, and aspects of treatment of importance to patients. The information obtained in this way will be useful to inform patient-centric study design as well as to evaluate change over time in patient perceptions.

Limitations of this analysis include the small sample number and potential for interpretation errors resulting from cultural and language differences in the study population. Due to the voluntary nature of the SAT, responders were self-selected. Additionally, there was no cognitive debriefing of study participants to elicit feedback regarding the relevance of the study question and their understanding of the question. The selected approach for this study also limits the ability and utility of quantifying the results of the natural language processing analyses.

\section{Conclusion}

This study demonstrates the feasibility and utility of using NLP of patient free-text responses to open questions to capture patient experience of CRSwNP and its treatment in 
their own voices. NLP of free-text responses provides qualitative data, which are not only useful in terms of "bedside feedback" in the specific population studied during the trial, but introduces an element of "narrative medicine" to discover perspectives that are important to the patient and should therefore be a focus of treatment. The results provide support for this approach to identify the most relevant concepts and related outcome measures for use in clinical trials of treatments. The present study found that "smell" was among the most frequently used words in patient free-text responses, highlighting sense of smell as particularly important to patients and identifying this as an important efficacy outcome measure in the context of future clinical trials.

\section{Abbreviations}

CRSwNP, chronic rhinosinusitis with nasal polyps; CT, computed tomography; HRQoL, health-related quality of life; IL, interleukin; MFNS, mometasone furoate nasal spray; NLP, natural language processing; NP, nasal polyp; PRO, patient-reported outcome; SAT, selfassessment of treatment; SD, standard deviation; SNOT22, 22-item Sino-Nasal Outcome Test; UPSIT, University of Pennsylvania Smell Identification Test; VAS, Visual Analog Scale.

\section{Data Sharing Statement}

Qualified researchers may request access to patient-level data and related study documents, including the clinical study report, study protocol with any amendments, blank case report form, statistical analysis plan, and dataset specifications. Patient-level data will be anonymized and study documents will be redacted to protect the privacy of our trial participants. Further details on Sanofi's datasharing criteria, eligible studies, and process for requesting access can be found at: http://www.clinicalstudydatare quest.com/.

\section{Ethical Approval}

The study was designed and conducted in accordance with the Declaration of Helsinki. The trial protocol was approved by the applicable institutional review board for each study site: UZ Gent Commissie voor Medische Ethiek, Gent, Belgium; CEIC Hospital Clínic i Provincial, Barcelona, Spain; Regionala etikprövningsnämnden i Stockholm, Sweden; Partners Human Research Committee, Boston, MA, USA; and Schulman Associates Institutional Review Board, Cincinnati, OH, USA.

\section{Consent to Participate}

All patients provided written informed consent to participate in the trial, and a written informed consent was signed by the participants specifically for the SAT tool, including permission to publish results (ie answers) and analyses of the questionnaires.

\section{Consent for Publication}

All authors and persons named in acknowledgments consented to publication.

\section{Acknowledgments}

The authors thank Jérôme Msihid for statistical analysis, Neil Graham for comments and insights, Nora Crikelair and Diane Barry for organizational and editorial assistance, and Maggie Tarrio Watson and Matt Lewis for medical writing assistance.

\section{Author Contributions}

Asif H. Khan, Bruno Falissard, Paulo Carita, Leda P Mannent, Gianluca Pirozzi, and Laurent Eckert contributed to the conception and design of the study, and data analysis and interpretation. Adeline Abbe, Matthew Reaney, Jingdong Chao, Nikhil Amin, and Puneet Mahajan contributed to data analysis and interpretation. Claus Bachert and Joaquim Mullol contributed to the conception and design of the study, data acquisition, and data analysis and interpretation. All authors contributed to drafting or revising the article, have agreed on the journal to which the article will be submitted, gave final approval of the version to be published, and agree to be accountable for all aspects of the work.

\section{Funding}

This research was sponsored by Sanofi and Regeneron Pharmaceuticals, Inc. ClinicalTrials.gov identifier: NCT01920893. Medical writing and editorial assistance provided by Maggie Tarrio Watson, PhD, of Excerpta Medica, and Matt Lewis, PhD, of Adelphi Communications, Macclesfield, UK, funded by Sanofi Genzyme and Regeneron Pharmaceuticals, Inc.

\section{Disclosure}

AHK, PC, LPM, PM, and LE are employees of Sanofi, and may hold stock and/or stock options in the company. AA, MR, and GP are former employees of Sanofi. JC and NA are employees of Regeneron Pharmaceuticals, Inc. and shareholders in the company. BF is a consultant for 
AbbVie, Actelion, Allergan, Almirall, Alnylam, Amgen, Astellas, AstraZeneca, Bayer, Biocodex, Biogen, Biopecs, Bioprojet, Biotronik, BMS, Boehringer Ingelheim, Celgène, Chiesi, D\&A Pharma, Daiichi-Sankyo, Eisai, Eli Lilly, Ethypharm, Genevrier, Genzyme, Gilead, Grunenthal, GSK, HRA, IDM Pharma, Idorsia, Indivior, Janssen, Lundbeck, Léo, Menarini, Novonordisk, MSD, Novartis, Otsuka, Pfizer, Pierre Fabre, Recordati, Roche, Sanofi, Servier, Stallergenes Greer, Takeda, UCB, and ViiV. CB is an advisory board member for ALK, ASIT Biotech, AstraZeneca, Intrexon Actobiotics, Novartis, Sanofi, and Stallergenes Greer. JM is a member of national or international advisory boards, and received speaker fees or funding for clinical trials and research projects from ALK, AstraZeneca, Genentech, GSK, Glenmark, Menarini, Mitsubishi-Tanabe, MSD, Mylan-MEDA Pharma, Novartis, Regeneron Pharmaceuticals, Inc. Sanofi Genzyme, UCB Pharma, and Uriach Group. The authors report no other conflicts of interest in this work.

\section{References}

1. Halpern J. From Detached Concern to Empathy: Humanizing Medical Practice. New York, NY: Oxford University Press; 2001.

2. Greenhalgh T, Hurwitz B. Narrative based medicine: why study narrative? $\quad B M J . \quad 1999 ; 318(7175): 48-50 . \quad$ doi:10.1136/bmj.31 8.7175 .48

3. Doward LC, Gnanasakthy A, Baker MG. Patient reported outcomes: looking beyond the label claim. Health Qual Life Outcomes. 2010;8:89. doi:10.1186/1477-7525-8-89

4. Hareendran A, Gnanasakthy A, Winnette R, Revicki D. Capturing patients' perspectives of treatment in clinical trials/drug development Contemp Clin Trials. 2012;33(1):23-28. doi:10.1016/j.cct.20 11.09.015

5. Messina J, Grainger DL. A pilot study to identify areas for further improvements in patient and public involvement in health technology assessments for medicines. Patient. 2012;5(3):199-211.

6. Wagland R, Recio-Saucedo A, Simon M, et al. Development and testing of a text-mining approach to analyse patients' comments on their experiences of colorectal cancer care. BMJ Qual Saf. 2016;25 (8):604-614. doi:10.1136/bmjqs-2015-004063

7. Weaver M, Patrick DL, Markson LE, et al. Issues in the measurement of satisfaction with treatment. Am J Manag Care. 1997;3(4):579-594.

8. Regnault A, Balp MM, Kulich K, Viala-Danten M. Validation of the treatment satisfaction questionnaire for medication in patients with cystic fibrosis. J Cyst Fibros. 2012;11(6):494-501. doi:10.1016/j. jcf.2012.04.007

9. Reaney M, Bush E, New M, et al. The potential role of individual-level benefit-risk assessment in treatment decision making: a DIA study endpoints community workstream. Ther Innov Regul Sci. 2019;53(5):630-638. doi:10.1177/2168479018807448

10. Edwards P. Questionnaires in clinical trials: guidelines for optimal design and administration. Trials. 2010;11:2. doi:10.1186/1745-6215$11-2$
11. Falissard B, Simpson EL, Guttman-Yassky E, et al. Qualitative assessment of adult patients' perception of atopic dermatitis using natural language processing analysis in a cross-sectional study. Dermatol Ther (Heidelb). 2020;10(2):297-305. doi:10.1007/s13555020-00356-0

12. Abbe A, Grouin C, Zweigenbaum P, Falissard B. Text mining applications in psychiatry: a systematic literature review. Int $J$ Methods Psychiatr Res. 2016;25(2):86-100. doi:10.1002/mpr.1481

13. Fokkens WJ, Lund VJ, Hopkins C, et al. European position paper on rhinosinusitis and nasal polyps 2020. Rhinology. 2020;58(Suppl S29):1-464. doi:10.4193/Rhin20.401

14. Khan A, Huynh TMT, Vandeplas G, et al. The GALEN rhinosinusitis cohort: chronic rhinosinusitis with nasal polyps affects health-related quality of life. Rhinology. 2019;57(5):343-351.

15. Palmer JN, Messina JC, Biletch R, et al. A cross-sectional, population-based survey of US adults with symptoms of chronic rhinosinusitis. Allergy Asthma Proc. 2019;40(1):48-56. doi:10.2500/ aap.2019.40.4182

16. Bhattacharyya N, Villeneuve S, Joish VN, et al. Cost burden and resource utilization in patients with chronic rhinosinusitis and nasal polyps. Laryngoscope. 2019;129(9):1969-1975. doi:10.1002/ lary. 27852

17. Bachert C, Bhattacharyya N, Desrosiers M, Khan AH. Burden of disease in chronic rhinosinusitis with nasal polyps. $J$ Asthma Allergy. 2021;14:127-134. doi:10.2147/JAA.S290424

18. Oyer SL, Mulligan JK, Psaltis AJ, et al. Cytokine correlation between sinus tissue and nasal secretions among chronic rhinosinusitis and controls. Laryngoscope. 2013;123(12):E72-E78. doi:10.1002/ lary. 24305

19. Miljkovic D, Bassiouni A, Cooksley C, et al. Association between group 2 innate lymphoid cells enrichment, nasal polyps and allergy in chronic rhinosinusitis. Allergy. 2014;69(9):1154-1161. doi:10.1111/ all. 12440

20. Macdonald LE, Karow M, Stevens S, et al. Precise and in situ genetic humanization of $6 \mathrm{Mb}$ of mouse immunoglobulin genes. Proc Natl Acad Sci U S A. 2014;111(14):5147-5152. doi:10.1073/ pnas. 1323896111

21. Murphy AJ, Macdonald LE, Stevens S, et al. Mice with megabase humanization of their immunoglobulin genes generate antibodies as efficiently as normal mice. Proc Natl Acad Sci U S A. 2014;111 (14):5153. doi:10.1073/pnas.1324022111

22. Gandhi NA, Pirozzi G, Graham NMH. Commonality of the IL-4/IL13 pathway in atopic diseases. Expert Rev Clin Immunol. 2017;13 (5):425-437. doi:10.1080/1744666XX.2017.1298443

23. DUPIXENT ${ }^{\circledR} \quad$ (dupilumab) injection [prescribing information]. Bridgewater, NJ; Tarrytown, NY: Sanofi-Aventis U.S. LLC; Regeneron Pharmaceuticals, Inc.; 2019.

24. DUPIXENT ${ }^{\circledR}$ (dupilumab) injection [summary of product characteristics]. Paris, France: Sanofi-Aventis Groupe; 2017.

25. Bachert C, Mannent L, Naclerio RM, et al. Effect of subcutaneous dupilumab on nasal polyp burden in patients with chronic sinusitis and nasal polyposis: a randomized clinical trial. JAMA. 2016;315 (5):469-479. doi:10.1001/jama.2015.19330

26. Bachert C, Han JK, Desrosiers M, et al. Efficacy and safety of dupilumab in patients with severe chronic rhinosinusitis with nasal polyps (LIBERTY NP SINUS-24 and LIBERTY NP SINUS-52): results from two multicentre, randomised, double-blind, placebo-controlled, parallel-group Phase 3 trials. Lancet. 2019;394 (10209):1638-1650. doi:10.1016/S0140-6736(19)31881-1

27. Bachert C, Hellings PW, Mullol J, et al. Dupilumab improves health-related quality of life in patients with chronic rhinosinusitis with nasal polyposis. Allergy. 2020;75(1):148-157. doi:10.1111/all.13984 
28. Locke S, Bashall A, Al-Adely S, et al. Natural language processing in medicine: a review. Trends Anaesth Crit Care. 2021;38:4-9. doi:10.1016/j.tacc.2021.02.007

29. de Hollander G, Marx M. Summarization of meetings using word clouds. In computer science and software engineering (CSSE). Paper presented at: CSI International Symposium 2011. IEEE; 2011:54-61.

30. Newman ME. Fast algorithm for detecting community structure in networks. Phys Rev E Stat Nonlin Soft Matter Phys. 2004;69(6 Pt 2):066133. doi:10.1103/PhysRevE.69.066133

31. Clauset A, Newman MEJ, Moore C. Finding community structure in very large networks. Phys Rev E. 2004;70(6):066111. doi:10.1103/ PhysRevE.70.066111

32. Levy JM, Mace JC, Bodner TE, et al. Defining the minimal clinically important difference for olfactory outcomes in the surgical treatment of chronic rhinosinusitis. Int Forum Allergy Rhinol. 2017;7 (8):821-826. doi:10.1002/alr.21964

33. Sindwani R, Han JK, Soteres DF, et al. Navigate i: randomized, placebo-controlled, double-blind trial of the exhalation delivery system with fluticasone for chronic rhinosinusitis with nasal polyps. $\mathrm{Am}$ J Rhinol Allergy. 2019;33(1):69-82. doi:10.1177/1945892418810281

34. Ricci-Cabello I, Saletti-Cuesta L, Slight SP, Valderas JM. Identifying patient-centred recommendations for improving patient safety in general practices in England: a qualitative content analysis of free-text responses using the patient reported experiences and outcomes of safety in primary care (PREOS-PC) questionnaire. Health Expect. 2017;20(5):961-972.

35. Vehviläinen-Julkunen K, Turpeinen S, Kvist T, et al. Experience of ambulatory cancer care: understanding patients' perspectives of quality using sentiment analysis. Cancer Nurs. 2021;44(6):E331-8. doi:10.1097/NCC.0000000000000845
36. Shu CH, Lee PO, Lan MY, Lee YL. Factors affecting the impact of olfactory loss on the quality of life and emotional coping ability. Rhinology. 2011;49(3):337-341. doi:10.4193/Rhino10.130

37. Alt JA, Mace JC, Buniel MC, et al. Predictors of olfactory dysfunction in rhinosinusitis using the brief smell identification test. Laryngoscope. 2014;124(7):E259-E266. doi:10.1002/ lary. 24587

38. Mattos JL, Schlosser RJ, Storck KA, Soler ZM. Understanding the relationship between olfactory-specific quality of life, objective olfactory loss, and patient factors in chronic rhinosinusitis. Int Forum Allergy Rhinol. 2017;7(7):734-740. doi:10.1002/ alr. 21940

39. O'Cathain A, Thomas KJ. "Any other comments?" Open questions on questionnaires - A bane or a bonus to research? BMC Med Res Methodol. 2004;4:25. doi:10.1186/1471-2288-4-25

40. Greaves F, Ramirez-Cano D, Millett C, et al. Use of sentiment analysis for capturing patient experience from free-text comments posted online. J Med Internet Res. 2013;15(11):e239. doi:10.2196/ jmir.2721

41. Dambha-Miller H, Silarova B, Irving G, et al. Patients' views on interactions with practitioners for type 2 diabetes: a longitudinal qualitative study in primary care over 10 years. $\mathrm{Br} J$ Gen Pract. 2018;68(666):e36-e43. doi:10.3399/bjgp17X693917
Patient Preference and Adherence

\section{Publish your work in this journal}

Patient Preference and Adherence is an international, peer-reviewed, open access journal that focusing on the growing importance of patient preference and adherence throughout the therapeutic continuum. Patient satisfaction, acceptability, quality of life, compliance, persistence and their role in developing new therapeutic modalities and compounds to optimize clinical outcomes for existing disease

\section{Dovepress}

states are major areas of interest for the journal. This journal has been accepted for indexing on PubMed Central. The manuscript management system is completely online and includes a very quick and fair peer-review system, which is all easy to use. Visit http:// www.dovepress.com/testimonials.php to read real quotes from published authors. 